\title{
¿CÓMO GERENCIAR UN PROYECTO SOCIAL A TRAVÉS DE LA MATRIZ VESTER EN PLANIFICACIÓN ESTRATÉGICA? CASO: EXPLOTACIÓN MINERA EN TIMBIQUÍ (CAUCA) ${ }^{1}$
}

\author{
How to manage a social project through a Vester matrix in strategic planning \\ Case: mining exploitation in Timbiqui (Cauca)
}

\author{
Víctor José Rodríguez Restrepo ${ }^{2}$ \\ vrodriguezrestrepo@outlook.com
}

Universidad de La Salle

Bogotá, Colombia

\begin{abstract}
Resumen
El trabajo de investigación es la representación de una problemática vigente en el Departamento del Cauca con su población afro-indígena-campesina. Esta población se ha visto afectada por la escasa oportunidad laboral y es aprovechada por los diferentes grupos al margen de la ley en la explotación de la minería ilegal. El trabajo tiene por objetivo mostrar a través de la aplicación de la matriz de Vester, herramienta de la planificación estratégica, que tan posible es determinar el problema central, sus causas y efectos sobre el manejo inadecuado de los recursos naturales, quiénes son los protagonistas del desequilibrio que se registra en la región. Finalmente se plantea unas posibles alternativas de acción por parte de los interesados para corregir el problema que perjudica a sus pobladores y al manejo de los recursos naturales así: i) La legalización, control y apoyo tecnológico por parte del estado para reglamentar la minería artesanal, ii) capacitación y formalización del personal adecuado con las competencias para la explotación minera controlada y iii) exigir a las compañías mineras extranjeras un mayor aporte en impuestos y responsabilidad social para el país.

Un directivo que trabaja la planificación estratégica en el campo de lo social debe orientar su toma de decisiones hacia el uso y la aplicación de las competencias directivas para llevar a la ejecución un plan de acción en donde se permita ejercer la justicia, las normas ambientales y la condición legal del trabajo de sus habitantes y gobernantes. Para evidenciar lo anterior, se describe el caso: Explotación minera en Timbiquí (Cauca).

El documento se inicia presentando los aspectos teóricos de la planificación estratégica y termina con un ejercicio práctico de la situación.
\end{abstract}

Palabras clave: Solución de problemas; factores claves de éxito; matriz de Vester; toma de decisiones gerenciales.

\footnotetext{
${ }^{1}$ Cite este artículo como: Rodríguez-Restrepo, V (2020). ¿Cómo gerenciar un proyecto social a través de la matriz Vester en planificación estratégica? Caso: explotación Minera en Timbiquí, Punto de vista, 11 (17)., pp. 63-84.

${ }^{2}$ Administrador de empresas de la Universidad Externado de Colombia; especialista en Gerencia de Talento Humano de la Escuela de Administración y Negocios EAN. Profesor Investigador adscrito a la línea de investigación GESOE (Gestión, Organización y Emprendimiento) de la Universidad De la Salle. Correo electrónico: vrodriguezrestrepo@outlook.com.
} 


\begin{abstract}
The research work is the representation of a current problem with Afro-indigenous-peasant population in the Department of Cauca. Those people are being affected by the scarce job opportunity and taken advantage of by different groups outside the law in the exploitation of illegal mining. Its objective is to show through the application of the Vester matrix, a strategic planning tool, if it is possible to determine the central problem, its causes and effects on the inadequate management of natural resources, who are the protagonists of the imbalance of the people who live and take advantage of the region. Finally, it discusses some possible alternatives for action by the interested parties to correct the problem that harms its inhabitants and the management of natural resources as follows: i) the legalization, control and technological support by the state to regulate artisanal mining, ii) training and formalization of the appropriate personnel with the competencies for controlled mining exploitation and, iii) demand from foreign mining companies a greater contribution in taxes and social responsibility for the country.

A manager who works on strategic planning in the social field must guide his decision-making towards the use and application of managerial skills to carry out an action plan where justice, environmental regulations and the legal status of the work of its inhabitants and rulers. To demonstrate the above, the case is described: Mining exploitation in Timbiqui (Cauca).

The document begins by presenting the theoretical aspects of strategic planning and ends with a practical exercise on the situation.
\end{abstract}

Key words:

Problem solving; key success factors; Vester matrix; Management decision making.

$\begin{array}{ll}\text { Recepción: } & 18.03 .2020 \\ \text { Aceptación: } & 26.10 .2020\end{array}$

\title{
INTRODUCCIÓN
}

\section{LA PLANIFICACIÓN ESTRATÉGICA}

Las empresas están expuestas a los cambios continuos del entorno. De ahí la importancia de la planificación estratégica que se define como: "La identificación sistemática de las oportunidades y peligros que surgen en el futuro, los cuales combinados con otros datos importantes proporcionan la base para que una empresa tome las mejores decisiones en el presente para explotar las oportunidades y evitar los peligros." (Steiner, 1997; pág 20).

Es importante anotar que la toma de decisión gerencial va a estar influenciada por el conocimiento alcanzado por parte de los gerentes, acerca de cómo aplicar una, dos herramientas, o las más adecuadas, para el análisis externo, pero de acuerdo o conforme a la información obtenida y examinada para resolver los problemas que a diario se presentan en las empresas. Cuando hablamos de planificar el trabajo de los demás, los problemas obedecerán en su gran medida a las señales arrojadas por el entorno y específicamente, del subsector al cual pertenece la empresa que monitorea los datos del PESTAL (las políticas del sector o del gremio, la economía de país; lo sociocultural; la tecnología utilizada; los aspectos ambientales y su normatividad vigente y lo legal) 
(Aguilar, 2020). Algunas señales emitidas en el ambiente tendrán una sensación de amenazas y otras de oportunidades algunas conocidas, otras no, algunas pueden pronosticarse por su ímpetu en el contexto, otras, son el resultado de los cambios socio-políticos previstos por ciertos gobernantes de naciones, con innegable poderío económico - decisorio, para establecer planes con capacidad de respuesta mundial, en algunos casos inmediata, en otros casos no, pero se presentan en muchos aspectos como sorpresas para los menos interesados.

Cuando se realiza un análisis externo sobre un sector en particular, el PESTAL puede arrogar situaciones favorables o desfavorables que ayudan o deterioran las condiciones propias del entorno, llegando a ocasionar problemas a las empresas estudiadas. De ahí la existencia de varios tipos de problemas se muestra con algunos ejemplos a saber:

\section{Los problemas en la Planificación estratégica:}

Todo equipo gerencial de planificación estratégica debe considerar los siguientes problemas, sobre los cuales deberá ejercer acciones pertinentes y conducentes a buscar soluciones apropiadas, ellos son:

- Problemas muy urgentes con efecto amplio y que requieren atención inmediata. Como ejemplo tenemos: el caso del cambio climático de la tierra. Para una empresa dedicada a la exportación de flores, en la siembra se esperan lluvias moderadas, pero llega el efecto del niño y quema las mieses de las flores ocasionando pérdidas e incumplimiento en las entregas internacionales.

- Problemas urgentes en forma moderada con un efecto amplio y que requieren atención inmediata. Como ejemplo se tiene el siguiente: Los incrementos en los costos del sistema logístico de transporte y empacado del producto resultan de difícil manejo, cuando la empresa no posee la tecnología ni los recursos que le permita hacer uso de las entregas del producto, de acuerdo con una programación de producción y despachos oportuno.

- Problemas que no son urgentes, que producen un efecto amplio y que requieren una vigilancia continua. Como caso se enuncia la siguiente situación: En una empresa fabricante de grifos, se presentan imperfectos en la fabricación del producto que sobrepasan el $15 \%$ de la programación mensual establecida, sin superar el porcentaje de demanda.

- Problemas que se constituyen en falsas alarmas y que pueden dejar de tenerse en consideración. La competencia quiere sustraer al personal de ventas de la compañía, con mejores salarios y compensaciones se escuchan rumores de algunos proveedores.

"Los problemas urgentes se asignan por la gerencia para su estudio y solución a las unidades de la empresa encargadas para atender la situación. La alta dirección vigila el proceso de solución de problemas a través de las estrategias y tácticas" para su implementación, en otras palabras, realiza lo importante de la gestión (Adaptado de Anssof, 1997, pág 13 - 17).

\section{Los recursos para la planificación estratégica:}

Todo gerente debe planificar de acuerdo con como formule la estrategia para la organización, tendrá en cuenta los recursos con los cuales ejercerá su plan de acción, ellos son:

- Recursos Físicos: lo tangible máquinas, locales, materias primas, vehículos, herramientas, equipos, etc.

- Recursos Económicos: son los que posee la empresa en dineros, capital social o en algunos casos se deberá hacer uso de la solicitud del crédito para los logros pretendidos.

- Recursos Técnicos y/o Tecnológicos: Representados en conocimientos de los procesos, máquinas, equipos, software especializado o la medida, formas de operar en campos del conocimiento científico y experimental), la mecanización, automatización, la electrónica, la robótica y en casos especiales la nanotecnología. 
- Recursos Mercadológicos: Son los que la organización ofrece a sus clientes bien como un producto o como un servicio, en muchos casos con innovación y creatividad y, en otros, con una propuesta de valor reconocible y diferenciado.

- Recursos de personal y Capital humano: Entendidos como el personal necesario para desarrollar la gestión gerencial, serán recursos cuando las personas son necesarias para cumplir un fin y no se genera en ellas expectativas de crecimiento personal y organizacional. Serán talentos cuando el capital intelectual es la posesión de conocimientos, experiencia aplicada, tecnología organizacional, relaciones con clientes y destrezas profesionales que dan a una organización una ventaja competitiva en el mercado.

- Recursos Naturales: Se clasifican en renovables y no renovables, los renovables son aquellos que no debemos dejar agotar, proteger y mantener unos son insumos y otros como las materias primas reciben transformación primaria para ser convertidos en sub-productos y materiales para darle continuidad a los procesos productivos.

- Recursos Administrativos: Ellos son: La planeación, organización, dirección y control se consideran las funciones administrativas con las cuales un gerente realiza su plan de ejecución organizacional.

Los gerentes deben poseer entre otras competencias las siguientes: "Orientación ambiental" "Demostrar sensibilidad hacia los desarrollos sociales, económicos y políticos y otros factores ambientales que puedan, presumiblemente, afectar al trabajo o a la organización". De igual forma la competencia de "Planificación y organización" "determina eficazmente las metas y prioridades estipulando la acción, los plazos y los recursos requeridos para alcanzarlas". En consecuencia, "El sentido de urgencia" "Percibir la exigencia real de determinadas tareas y actuar de manera firme para alcanzar su realización en plazos muy breves de tiempo." Orientación al logro "Determinación para fijar las propias metas de forma ambiciosa, por encima de los estándares y de las expectativas, mostrando insatisfacción con el desempeño medio" "Visión." Pensar sobre la base de la estrategia de la empresa y convertirla en objetivos concretos para el propio campo de responsabilidad, ser capaz de hacer contribuciones a la formulación de la estrategia de la organización. (Cao, 1996), página 171216).

\section{Las habilidades de la gestión gerencial:}

Todo gerente de empresa sin importar su tamaño requiere de unas dimensiones básicas para su ejercicio ellas son:

- Habilidad cognitiva: Entendida como saber categorizar problemas administrativos y ver las relaciones existentes entre las categorías. Comprender lo particular a través del conocimiento de lo general. Su objetivo es formular políticas, desarrollar procesos y diseñar estructuras.

- Habilidad analítica: saber la utilidad y el potencial de las técnicas administrativas y adquirir más realismo, profundidad y creatividad en la solución de problemas. Su objetivo es aprender a descomponer problemas, identificar variables y establecer relaciones causa-efecto.

- Habilidad de Comportamiento: son las actitudes para obtener respuestas de comportamiento coherentes con los objetivos de eficiencia, eficacia, satisfacción, efectividad y seguridad en el trabajo. Su objetivo es encontrar nuevas formas de comunicación y de interacción de equipo.

- Habilidad de Acción: es el desarrollo de la capacidad interior para transformar objetivos, valores, conocimientos en formas efectivas de acción. Su objetivo es aprender acerca de sí mismo, desarrollar compromisos con la misión de la empresa. Un gerente efectivo es aquel que lleva los objetivos de la organización a feliz término, tiende a conducir la organización por el camino accesible donde fluye la comunicación de manera directa con el equipo de trabajo, las responsabilidades son compartidas por el grupo de personas que tiene a cargo la dirección de la empresa, la coordinación se hace en función de la claridad existente de los diferentes cargos establecidos en la estructura organizacional de la empresa. 
Las competencias directivas

Más que habilidades gerenciales, aparecen otras llamadas las competencias para el gerente y ellas deben ser atendidas por la experiencia directiva expresada en muchos casos, se muestran en la presente tabla.

\begin{tabular}{|c|c|c|c|c|}
\hline $\begin{array}{l}\text { 1. Liderazgo: Capacidad } \\
\text { de un directivo para } \\
\text { influir en otras } \\
\text { personalidades de la } \\
\text { empresa y para } \\
\text { orientar el curso de } \\
\text { acción hacia los } \\
\text { objetivos del colectivo } \\
\text { humano. }\end{array}$ & $\begin{array}{l}\text { 2. Dirigir escuchando: } \\
\text { Es más importante } \\
\text { escuchar que hablar. } \\
\text { Debe haber } \\
\text { predisposición a } \\
\text { prestarles atención a } \\
\text { los subordinados, ya } \\
\text { que ellos recibirán } \\
\text { órdenes } \\
\text { instrucciones. }\end{array}$ & $\begin{array}{l}\text { 3. Llegar a la gente } \\
\text { con empatía: } \\
\text { Ponerse en los pies } \\
\text { de la otra persona. } \\
\text { Si yo fuera...... ¿qué } \\
\text { haría...? estimular } \\
\text { la motivación de los } \\
\text { empleados. }\end{array}$ & $\begin{array}{l}\text { 4. Atender la } \\
\text { consonancia cognitiva: } \\
\text { lo que se piensa se dice } \\
\text { y se hace, de igual } \\
\text { forma se manifiesta } \\
\text { con acciones y } \\
\text { comportamientos. }\end{array}$ & $\begin{array}{l}\text { 5. Entregar informes de } \\
\text { acuerdo a los temas } \\
\text { tratados. } \\
\text { Informes: técnicos; } \\
\text { rutinarios; ejecutivos: } \\
\text { fiscales y de auditorías. }\end{array}$ \\
\hline $\begin{array}{lr}6 . & \text { Comunicación } \\
\text { asertiva: Hablar de } \\
\text { manera } & \text { puntual, } \\
\text { preocuparse } & \text { por } \\
\text { determinar que su } \\
\text { interlocutor entienda } \\
\text { el mensaje. }\end{array}$ & $\begin{array}{l}\text { 7. Escoja las personas } \\
\text { adecuadas para } \\
\text { delegar: Que } \\
\text { personas presentan } \\
\text { formación técnica y } \\
\text { cuales otras son } \\
\text { profesionales. Trate } \\
\text { de identificar } \\
\text { cualidades de la } \\
\text { gente. Trabaje el } \\
\text { Mentoring. }\end{array}$ & $\begin{array}{l}\text { 8. Prepare su } \\
\text { gente: Es } \\
\text { importante dar } \\
\text { confianza para } \\
\text { recibir confianza, } \\
\text { establezca las } \\
\text { personas con las } \\
\text { cuales usted puede } \\
\text { compartir ideas, } \\
\text { trabajo, estudio y } \\
\text { distracción. }\end{array}$ & $\begin{array}{l}\text { 9. Conformar equipos } \\
\text { de trabajo: Analice } \\
\text { competencias de su } \\
\text { gente, delegue trabajo, } \\
\text { identifique } \\
\text { experiencias y } \\
\text { entregue } \\
\text { empowerment. }\end{array}$ & $\begin{array}{l}\text { 10.Cultivar una red de } \\
\text { contactos: } \\
\text { Atraer a la gente es } \\
\text { una cualidad positiva } \\
\text { propia de la confianza, } \\
\text { el respeto y la } \\
\text { disposición hacia el } \\
\text { compromiso } \\
\text { organizacional. Formar } \\
\text { redes de apoyo. }\end{array}$ \\
\hline $\begin{array}{l}\text { 11.Tomar decisiones } \\
\text { frente a los problemas } \\
\text { complejos } \\
\text { Los problemas con } \\
\text { poca información, } \\
\text { requieren de quien los } \\
\text { enfrenta trabajar } \\
\text { pensamiento complejo } \\
\text { y el apoyo de técnicas } \\
\text { especiales para su } \\
\text { solución. }\end{array}$ & $\begin{array}{l}\text { 12. Monitorear el } \\
\text { entorno: El estar } \\
\text { informado implica un } \\
\text { buen manejo de } \\
\text { datos actualizados. } \\
\text { Un buen análisis del } \\
\text { PESTAL aplicado al } \\
\text { subsector que se } \\
\text { desea estudiar, } \\
\text { permite lograr al } \\
\text { equipo directivo, } \\
\text { contar con los } \\
\text { argumentos } \\
\text { necesarios para } \\
\text { planificar de manera } \\
\text { exitosa }\end{array}$ & $\begin{array}{l}\text { 12.Capacidad de } \\
\text { negociación: Las } \\
\text { técnicas utilizadas } \\
\text { para negociar bien } \\
\text { sea con } \\
\text { proveedores; } \\
\text { distribuidores; } \\
\text { clientes; personal } \\
\text { de la empresa; } \\
\text { funcionarios del } \\
\text { estado; sector } \\
\text { financiero; demás } \\
\text { accionistas y demás } \\
\text { los denominados } \\
\text { stakeholders } \\
\text { siempre se } \\
\text { delimitan dentro } \\
\text { del gana gana. }\end{array}$ & $\begin{array}{l}\text { 13. Orientar el trabajo } \\
\text { asociativor entre } \\
\text { empresas interesadas: } \\
\text { Trabajar en función de } \\
\text { alianzas estratégicas } \\
\text { entre empresas } \\
\text { permite: controlar } \\
\text { gastos, recuperar los } \\
\text { costos previendo } \\
\text { inversiones, aprender } \\
\text { de los demás, visualizar } \\
\text { procesos eficientes sin } \\
\text { perder la razón social, } \\
\text { ni la independencia de } \\
\text { la firma. }\end{array}$ & $\begin{array}{l}\text { 14. Delegación de la } \\
\text { autoridad: transferir a } \\
\text { otro de manera } \\
\text { adecuada las tareas, } \\
\text { funciones y autoridad, } \\
\text { dándole la información } \\
\text { necesaria. Entregando } \\
\text { la capacidad para la } \\
\text { toma de decisiones en } \\
\text { el proceso de } \\
\text { cumplimento en } \\
\text { nombre de su propia } \\
\text { representación. }\end{array}$ \\
\hline
\end{tabular}

Tabla Nº ${ }^{\circ}$ Competencias directivas. Fuente: Adaptado de Paulo Roberto Motta (El arte de ser dirigente, pág. 28).

¿Por qué es importante planificar?

Se planifica por la necesidad de anticiparnos con buenas acciones ante los cambios imprevistos y previstos del entorno, para evitar futuras contingencias, para orientar a los departamentos de planeación de las empresas en donde se plantean ambiciosos planes de desarrollo sin entender las razones que van más allá de la capacidad analítica de quiénes lo conforman. Los principales aspectos a tener en cuenta en la planificación estratégica son:

1. Al realizar el examen a cerca de los beneficios en muchos casos resultan siendo inferiores ante los altos costos, la carencia de recursos, la incompetencia de las personas asignadas y la pérdida de tiempo. 
1. No se estudia claramente el entorno con sus fuerzas externas, dado que en muchos casos bloquean los caminos del proyecto, haciendo más difícil la labor de quiénes trabajan en la gerencia.

2. No se tiene claridad de los mecanismos de control que hacen parte de la planificación y más aún, cuando aparecen los problemas complejos es cuando surgen los imprevistos.

3. La información obtenida queda rápidamente desactualizada ante las lentas acciones de la gerencia para salirle al encuentro a los datos presentados.

4. La mínima preocupación por los interesados de un proyecto, ante los impactos que se pueden presentar al hacer mal uso de los recursos naturales afectando el medio ambiente.

5. En algunos casos se hacen cálculos inadecuados en la gestión financiera, cuando se entra en la ejecución de los planes, se queda corto el proyecto para poder operacionalizar.

6. Sirve para concientizar al equipo directivo de adoptar la innovación-creatividad como la aplicación de nuevas ideas para efectuar cambio bien en la empresa, el producto, el servicio, el proceso o un método de trabajo.

7. El desarrollo de una visión a largo plazo, con objetivos claros permitiendo acciones posibles de lograr, detectando suposiciones críticas para contrarrestarlas a través de estrategias orientadas hacia el crecimiento, sostenibilidad, competitividad y defensa según sea el caso.

Cuando se planifica es fundamental considerar un escenario prospectivo como lo sugiere Chung (2009) que argumenta: "Al hacer un plan estratégico prospectivo, si se tiene una visión estratégica prospectiva y unos planes estratégicos per se, no se debe olvidar en el modelamiento que las acciones tácticas deben estar enmarcadas en un pensamiento prospectivo, el cual es el plan operativo anual." (Antonieta, 2017, pp. 41-42). Para controlar y planificar de mejor forma, se deben tomar decisiones en donde se afecten de manera positiva el futuro de la organización, con base en el análisis de escenarios posibles y probables (Ver tabla 2).

\begin{tabular}{|cl|}
\hline Nivel prospectivo & Escenario estratégico para el año \\
\hline Nivel estratégico & Plan estratégico para el período \\
\hline Nivel de acciones estratégicas & Plan operativo anual \\
\hline Tabla $N^{\circ}{ }_{2}$ Niveles de planificación. Fuente: Chung, P. (2009) Prospectiva estratégica, más allá del plan estratégico. Revista facultad de ingeniería \\
Industrial 12(2); pág. 27-31.
\end{tabular}

¿Qué es un proyecto social?

Los proyectos sociales son aquellos en donde el Estado ha determinado mejorar las condiciones de vida de la población, debido a los fenómenos ocasionados por la naturaleza y los cambios climáticos, la protección al ecosistema y fomentar la educación para el logro de estos fines (Constitución Nacional de Colombia, 1991. Art. 79). Para llevar a cabo la ejecución de un proyecto de desarrollo social se requiere de la buena voluntad y actitud positiva de la comunidad afectada, con necesidades y expectativas en donde la iniciativa de la participación ciudadana o rural se haga presente, con el fin de atender las actividades planificadas, ejecutadas y supervisadas permitiendo de esta forma la reconversión de los problemas en soluciones valederas en el largo plazo.

De otra parte, se requiere de la comunidad la presencia de líderes que aporten ideas, planteen alternativas de solución, deseen trabajar conjuntamente de forma colaborativa a través del respeto mutuo, aceptando las diferencias de género, las circunstancias existentes del medio y, con tolerancia, proponer para salir adelante aunando esfuerzos. Para ello, se deberá responder los siguientes interrogantes como lo menciona Rafael Méndez en su texto: Formulación y Evaluación de Proyectos <enfoque para emprendedores> (Ajenjo, 2006, pag. 2-26).

- ¿Cuál es la población objetivo que se va a beneficiar con el proyecto?

- ¿Qué acciones se van a llevar a cabo? ¿Quiénes la realizarán, cómo se harán, cuándo y dónde?

- ¿Con qué recursos se cuenta para el proyecto y qué otros se necesitan? 
- ¿Cómo podemos obtener los recursos que no tenemos...?

- ¿Cómo se van a administrar estos recursos?

- ¿Cómo se van a evaluar los recursos del proyecto?

- ¿Cuáles indicadores de gestión que van a ser utilizados para el proyecto?

- ¿Qué relación beneficio/costo se evalúa frente al impacto social?

- ¿Qué factores condicionales se deben estudiar para la intervención del proyecto?

- ¿Cuáles son las necesidades y potencialidades de interés necesario?

¿Cómo se clasifican los proyectos?

El Instituto Latinoamericano de Política Económica y Social ILPES, entidad especializada en asocio con los gobiernos de los países latinoamericanos y las entidades internacionales que se ocupan de los problemas del desarrollo económico y social, preparan esta guía, para que sirva como instrumento de trabajo para aquellos investigadores y jefes de planes y programas en la elaboración de proyectos tendientes a resolver problemas sociales como instrumento de trabajo y orientación en la presentación de proyectos (ILPES, 2000). A continuación, se presenta una tabla adaptada sobre la clasificación y tipología de proyectos para Colombia.

\begin{tabular}{|c|c|c|}
\hline 1.PRODUCCIÓN DE BIENES & 2.PRODUCCIÓN DE SERVICIOS & $\begin{array}{l}\text { 3.INVESTIGACIÓN EN TECNOLOGÍAS - LÍNEAS DE } \\
\text { INVESTIGACIÓN DE PROGRAMAS }\end{array}$ \\
\hline $\begin{array}{l}\text { 1.1. Producción extractiva; } \\
\text { producción agrícola; crianza, } \\
\text { manutención animal y } \\
\text { sacrificio; reforestación } \\
\text { forestal. }\end{array}$ & $\begin{array}{l}\text { 2.1. Infraestructura física: } \\
\text { Transporte; comunicaciones; } \\
\text { Energía eléctrica; Saneamiento } \\
\text { básico; Urbanización }\end{array}$ & $\begin{array}{l}\text { Ley } 1530 \text { de } 2012 \text { Departamento Administrativo de } \\
\text { Ciencia y Tecnología e Innovación, Colciencias: Para } \\
\text { Investigación y Desarrollo: Innovación; Formación y } \\
\text { Capacitación }\end{array}$ \\
\hline \multirow[t]{3}{*}{$\begin{array}{l}\text { 1.2 Procesos de } \\
\text { Transformación: Bienes para } \\
\text { consumo final; intermedios } \\
\text { y de capital. }\end{array}$} & $\begin{array}{l}\text { 2.2. Infraestructura social: salud; } \\
\text { educación; vivienda y organización } \\
\text { social. }\end{array}$ & $\begin{array}{l}\text { Líneas de Investigación: } 1 \text {. Materiales, Minería y } \\
\text { Desarrollo de Energía; Ciencias Sociales y Humanas; } \\
\text { Seguridad y Defensa para la protección Nacional. } \\
\text { Telecomunicaciones e Informática. }\end{array}$ \\
\hline & $\begin{array}{l}\text { 2.3. Otros servicios: Profesionales; } \\
\text { Financieros; } \quad \text { Informativos; } \\
\text { distribución. }\end{array}$ & $\begin{array}{l}\text { 2. Agropecuarias y Desarrollo Rural; Agua y } \\
\text { Recursos naturales; Biodiversidad. }\end{array}$ \\
\hline & & $\begin{array}{l}\text { 3. Innovación en la transformación productiva y } \\
\text { social; formación de capacidades en } \mathrm{CT}+\mathrm{i} \text {; } \\
\text { Fortalecimiento en } \mathrm{SNCTI} \text {.) }\end{array}$ \\
\hline
\end{tabular}

Tabla 3. Clasificación de proyectos y líneas de investigación para Colombia. Fuente: Adaptado de la Clasificación proyectos ILPES (2000).

\section{MÉTODO}

\section{La matriz de Vester}

Es una técnica aplicada por Frederick Vester y que puede ser consultada en el siguiente enlace: (https://www.ingenioempresa.com/matrizdevester). Se entiende como una serie ordenada de filas y columnas en forma de matriz, donde se dan las posibles causas de una situación problema presentado en una comunidad, empresa, región, etc. Se dan unos criterios de calificación así:

\begin{tabular}{|ll|}
\hline 0 & No lo causa \\
\hline 1 & Lo causa indirectamente, su causalidad es muy débil \\
2 & Lo causa de forma semidirecta, su causalidad es media \\
3 & Lo causa directamente, su causalidad es fuerte \\
\hline
\end{tabular}


Para un análisis basado en la matriz de Vester se deben seguir los pasos que se describen a continuación: Determinar las variables problema al tratar de entender el problema central se encuentra que detrás de un problema existen otros, haciendo difícil el análisis de cuál de ellos será realmente el más importante. Redactar el problema analizarlo como un simple espectador, redactarlo en tercera persona para poderlo identificar claramente. Bautizar cada problema con un nomenclador así: Problema 1 - Problema 2 - Problema 3....... Sucesivamente. Ubicar todos los problemas encontrados en la matriz tanto de manera horizontal como vertical en orden homólogo. Calificar y valorar: Asigne las calificaciones comenzando por el problema 1 de la fila horizontal versus el problema 2 de la columna vertical, realizar la siguiente pregunta: ¿Qué tanto causa el problema 1 al problema 2? Establecer la relación de causalidad así:

- No lo causa

- Su causalidad es débil

- Su causalidad es media

- Su causalidad es fuerte

Finalmente, totalizar las filas (grado de incidencia y columnas grado de dependencia) la suma de cada fila será el total de la incidencia se conoce como incidencia/causa; también se conoce como fuerza motriz, porque ejerce influencia sobre los otros problemas. La suma de cada columna da el nivel de dependencia/efecto se le conoce como el nivel en el que un problema es causado por otros. Posteriormente, se debe realizar una gráfica problema donde el eje $X$ represente problemas activos: incidencia/causa y el eje $\mathrm{Y}$ represente problemas pasivos: dependencia/efecto. Clasificar los problemas: tomar el mayor valor total de la suma por filas y se divide por 2; tomar el mayor valor total de la suma por columna y se divide por 2; se obtiene cuatro (4) cuadrantes, a saber:

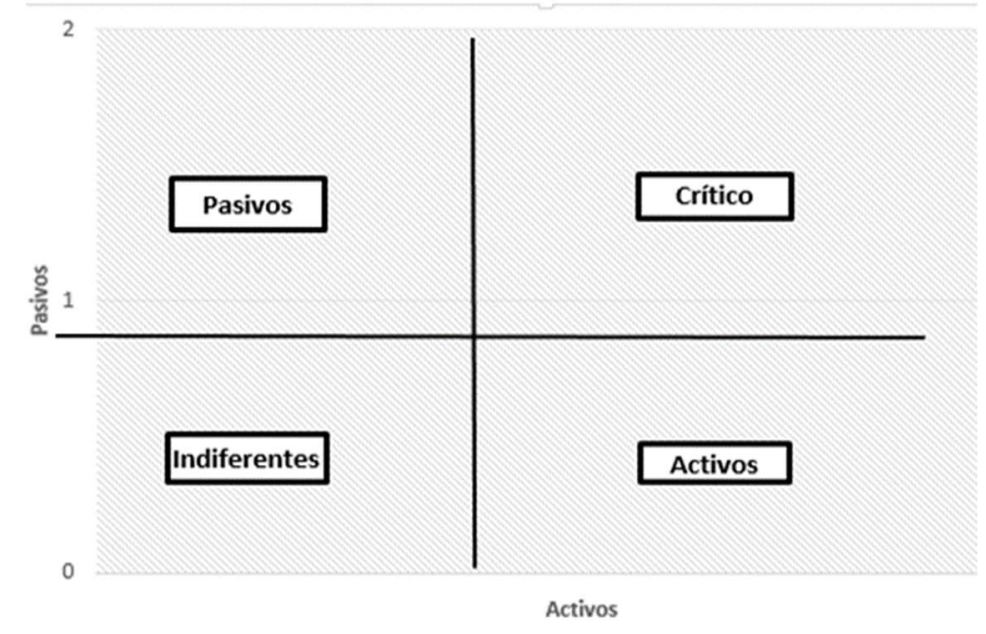

Figura 1. Áreas críticas del resultado de la Matriz de Vester. Fuente: Elaboración propia.

La clasificación resultante puede ser descrita como sigue:

- Problemas críticos: Total activos - pasivos altos causados por otros

- Problemas pasivos: Alto total pasivo y bajo total activo - poca incidencia causal

- Problemas indiferentes bajo total activos y pasivos (ni causan a otros ni son causados - baja prioridad 
- Problemas activos: Alto total de activos y bajo total pasivos no son causados por otros pero inciden mucho en otros criterios, requieren atención y manejo crucial es la causa principal de la situación problema.

\begin{tabular}{|c|c|c|c|c|c|c|c|c|}
\hline \multicolumn{8}{|c|}{ SITUACIÓN PROBLÉMICA A RESOLVER } & \multirow[b]{2}{*}{ INCIDENCIA } \\
\hline CÓDIGO & VARIABLE DE ESTUDIO & P1 & $\mathbf{P 2}$ & $P_{3}$ & P4 & $P_{5}$ & P6 & \\
\hline \multicolumn{9}{|l|}{ P1 } \\
\hline \multicolumn{9}{|l|}{ P2 } \\
\hline & DEPENDENCIA & & & & & & & \\
\hline
\end{tabular}

La matriz de Vester contribuye a diseñar el árbol problema (ver figura 2), técnica utilizada para identificar a través de múltiples causas previstas, en dónde es posible encontrar el problema principal o central. En las raíces se visualizan las causas del problema; el tronco representa el problema principal; en las hojas y ramas están los efectos o consecuencias. En un proyecto; caso de estudio o situación de momento de difícil análisis, en donde una comunidad emergente presenta una situación que la afecta, es posible con esta técnica realizar una investigación, para encauzar de forma apropiada la planificación estratégica. Existen otros elementos que intervienen en el respectivo análisis como la matriz de Vester; La matriz del marco Lógico; la matriz de los interesados, todos ellos son métodos que contribuyen a hacer más clara la solución de un problema como tal.

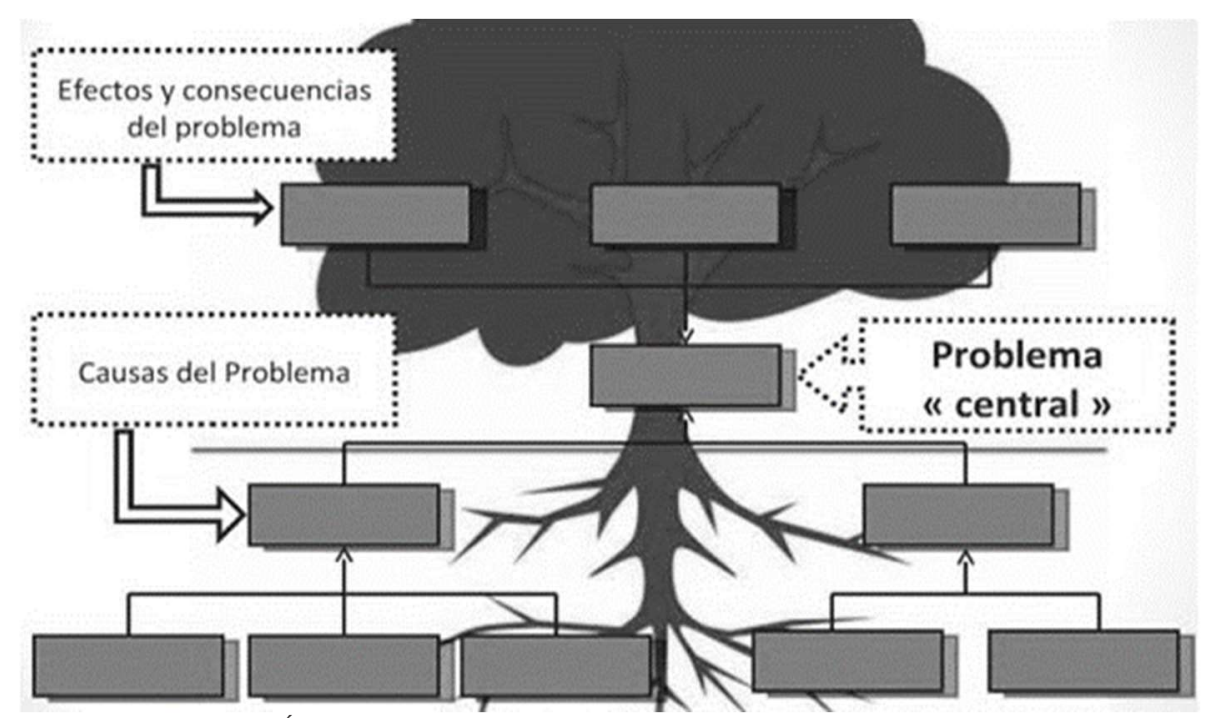

Figura 2. Árbol de problema. Fuente: http://organizadoresulta.blogspot.com.

El procedimiento continúa al elegir uno de los problemas ubicados en el cuadrante crítico (puntuación más alta en incidencia y dependencia). Los otros problemas encontrados en este cuadrante con puntajes más bajos serán las causas primarias al problema central elegido. Los problemas activos corresponden a causas secundarias 0 primarias, en caso de que existan pocos o tan sólo un problema crítico. Las consecuencias o efectos son los problemas denominados pasivos. 
Es fundamental para el éxito de un proyecto identificar a las personas interesadas desde el comienzo del mismo, así como analizar sus niveles de interés, expectativas, importancia e influencia en las acciones del mismo. Esta metodología se realiza para ir determinando la estrategia a través de las influencias positivas y mitigar los impactos. Se debe revisar periódicamente la matriz para ajustarla frente a los cambios eventuales, si se vienen involucrando actores al proyecto.

Los pasos para elaborarla son:

1. Identificar con claridad los stakeholders

2. Documentar para justificar sin son internos o externos y el nivel de las expectativas de cada uno de ellos.

3. Crear una matriz de doble entrada en donde el eje $\mathrm{Y}$ se denomina poderes y el eje $\mathrm{X}$ situar a los interesados (ver matriz de la siguiente hoja).

\begin{tabular}{|c|c|c|c|c|c|c|c|c|}
\hline \multicolumn{4}{|c|}{ MATRIZ DE LOS INTERESADOS } & \multicolumn{3}{|c|}{ TIPO DE ACTITUD } & \multirow[t]{2}{*}{ INTERÉS } & \multirow{2}{*}{$\begin{array}{l}\text { TIPO DE } \\
\text { PODER }\end{array}$} \\
\hline$N^{\circ}$ ORDEN & NOMBRE & $\begin{array}{l}\text { ROL EN EL } \\
\text { PROYECTO }\end{array}$ & $\begin{array}{l}\text { INFORMACIÓN } \\
\text { DE CONTACTO }\end{array}$ & 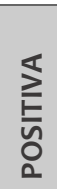 &  & 妾 & & \\
\hline
\end{tabular}

Tabla 5. Matriz de los interesados. Fuente: Tomado de http://www.ceolevel.com.

La información de la matriz de los interesados puede sistematizarse en un esquema como el que se presenta a continuación:

\begin{tabular}{|c|c|c|}
\hline \multirow{5}{*}{$\frac{\grave{d}}{0}$} & Latentes & Promotores \\
\hline & Mantenerlos satisfechos & Administrarlos con cercanía \\
\hline & Monitorear & Mantenerlos informados \\
\hline & Apáticos & Defensores \\
\hline & \multicolumn{2}{|c|}{ Interés } \\
\hline
\end{tabular}

Tabla 6. Síntesis Matriz de los interesados. Fuente: Tomado de http://www.ceolevel.com

Las personas y las entidades son las llamadas a hacer parte de los proyectos, en muchos casos, no solo el interés que los motiva, sino el ejercicio del poder bien sea económico; político, legal y, en algunos casos, el poder carismático que posee el líder de una comunidad. Lo importante, es conocer cuál va a ser su papel dentro del proyecto y si su actitud beneficia o no al estudio. Dentro de los posibles interesados en un proyecto se encuentran: El gobierno a través de sus representantes; las entidades públicas y privadas si presentan interés o se ven afectadas; las entidades internacionales si participan en el proyecto con dineros para el desarrollo del mismo; los líderes comunitarios; las personas interesadas en el proyecto y/o afectadas; los medios de comunicación por el interés de difusión; personas ajenas al proyecto, pero con intereses particulares. 


\section{CASO DE ESTUDIO: MINERÍA ILEGAL EN TIMBIQUÍ CAUCA}

El país se encuentra en medio de un auge minero - energético, las firmas transnacionales tienden a llegar con capital extranjero para la explotación de petróleo, carbón, minas de materiales poco conocidos como el torio, el coltán en la aplicación del hardware y en otros contextos tecnológicos como los celulares. El interés está en todo aquello que genere exploración y explotación de hidrocarburos para obtener energía.

La minería ilegal se ha convertido en uno de los mayores problemas de tipo social, ambiental y económico para las comunidades afro-indígenas y sus pobladores, en donde operan las firmas dedicadas a esta actividad. La falta de presencia del Estado en muchos de los territorios nacionales hace que los habitantes sean presas de las mafias organizadas para delinquir y sacar provecho de la explotación de la riqueza natural de varias de las regiones del país.

Las compañías extranjeras autorizadas por el gobierno nacional a través del Ministerio de Minas y Energía cuentan con toda la documentación al día, atienden las políticas ambientales y sociales, mostrando compromiso con los derechos humanos y con el entorno que los rodea. Sin embargo, la realidad es otra, mientras las comunidades enfrentan desastres sociales y ambientales producto de la extracción de los recursos, el beneficio real es para unos pocos pues los ingresos resultantes de dicha actividad no son oficiales, dado que no se reportan al Estado y no cumplen con los tributos legalmente previstos en la legislación nacional.

Un ejemplo interesante para analizar es el de la población de Timbiquí en el departamento del Cauca, municipio fundado en 1772 por Francisco Mosquera Andrés Saa. Los primeros pobladores fueron los indígenas embera wounaan desde este tiempo, los españoles los utilizaban para la explotación aurífera a las orillas de los ríos y quebradas. Su cultura se basa en tradiciones, costumbres ancestrales, el canto con sentimiento hereditario, tocan la marimba, bailan el currulao, el bunde y celebran la fiesta de los inocentes. Sus productos típicos son: el pescado, el guatín, el yuyo, la cocoroma y el chontaduro, consumen el dulce de miel caña blanca. "El municipio cuenta con 21.500 hab. Y conviven 28.000 personas con foráneos incluyendo los no nacionales atraídos por la minería ilegal”. (Radio Super, 2015)

La minería industrial o ilegal se apoya en gran medida con la financiación de grandes capitalistas o grupos al margen de la ley, ya sea, por cuestiones de seguridad, se respaldan en el apoyo permanente de los grupos armados ilegales como forma de garantía ante cualquier dificultad para enfrentar en el terreno de la explotación, mantener la población controlada, dominada y sometida a los lineamientos que determinan los dueños de la maquinaria, tecnología y demás, causando incertidumbre, generando inestabilidad al interior de las comunidades, cultura, identidad y vida colectiva (Banguera, 2017).

Se define minería artesanal o tradicional como "aquella que las comunidades han venido realizando durante décadas y hacen parte de sus prácticas culturales y medios de subsistencia para luchar contra la pobreza" (González, 2016, p. 1).

La minería ilegal tiene varias actividades como: la extracción, transporte y el procesamiento de materiales como petróleo crudo, gas natural, carbón mineral, minerales metalíferos y no metálicos; uranio, coltán y torio. La minería ilegal se convierte en un problema ambiental, normativo, económico, social y político. Como consecuencias de esta situación aparecen: la explotación inadecuada de recursos propios de la región, el alcoholismo, la prostitución, la ocupación laboral no reglamentada por organismo oficial, la inseguridad rural y ciudadana, los infantes no se educan, cuando llegan a las edades de juventud, son presas de las organizaciones que realizan prácticas ilegales.

En el sector ambiental, se da la explotación sin control de los recursos naturales afectando de manera negativa el ecosistema. Se estudian los siguientes efectos: 
1. Daño a la superficie terrestre, representada en un riesgo a la erosión muy alto afectando la biodiversidad, ocasionando mal manejo y contaminación de los ciclos de agua conmoviendo los procesos biológicos de flora y fauna. Al destruir la capa vegetal del suelo, los grandes movimientos de tierra se llevan los cultivos tradicionales sumado a los huecos con charcos en aguas muchas veces estancadas, presentan con el tiempo criaderos de zancudos, mosquitos, insectos transmisores de enfermedades contagiosas para la comunidad.

2. La liberación de sustancias tóxicas al extraer metales minerales, y elementos biológicos con ciertas concentraciones de microorganismos ácidos sulfúricos y óxidos de hierro, generan peligros para la salud humana. Con el pasar del tiempo, las personas siendo ya mayores comienzan a padecer las secuelas dejadas por el trabajo realizado en la juventud como consecuencia de la no prevención al manejo de estas sustancias contaminantes.

En los aspectos sociales se presentan también irregularidades afectando la ocupación de la gente en edad de trabajar, los grupos al margen de la ley ofrecen dádivas a la población del territorio, para contribuir en la explotación minera sin control de funcionarios del estado y la fuerza pública, causando incertidumbre en la comunidad ante la falacia de un negocio ilusorio, dejando como consecuencia, muchos casos de desatino y el desconcierto en la población juvenil. (Banguera A. B., 2017)

El corregimiento de San José de Timbiquí posee una temperatura de $28^{\circ}$ centígrados en medio de una inmensa variedad de flora y fauna rica en recursos naturales; el 90\% de la población se dedica a la explotación minera; las principales fuentes hídricas se encuentran contaminadas; la utilización de la energía eléctrica tan solo se tiene por 4 horas al día de 6.00 a 10.00 p.m. El 10\% restante de la población se dedica a la pesca, la agricultura; la caza; el trabajo con maderas y el comercio en pequeña escala. En el desarrollo del trabajo minero, la comunidad no cuenta con una planta eléctrica y servicio de agua potable las 24 horas del día, las escuelas y colegios no poseen tecnología blanda para un mejor proceso de formación de la primaria y secundaria; no se cuenta con un buen puesto de salud, que pueda atender tanta población; no hay emprendimientos en proyectos agrícolas, producto de la dirección y orientación del gobierno seccional. La mujer afrodescendiente ocupa una labor predominante y destacada en la sociedad familiar vela por los hijos en el cuidado de la alimentación y su crecimiento, realiza labores de pesca, en las minas tiene la función de apañar la tierra picada hasta los canelones, para ser lavada con agua seleccionando el metal aurífero, es considerada un soporte importante en la toma de decisiones del grupo familiar.

En el informe del Ministerio de Minas y Energía, páginas 17 y 18 (Ministerio de Minas y Energía, 2017) se pone en evidencia que gobierno central sigue empeñado en hacer de la locomotora minera un factor económico indispensable, para la economía del país atrayendo grandes capitales como inversión extranjera para la explotación de recursos, con el fin de mejorar la competitividad del país. Para ello, existen cinco (5) pilares estratégicos sobre los cuales el gobierno se apoya a saber:

- En lo político: El plan de desarrollo 2014-2018 habla de mejorar y crecer en el ámbito competitivo a través de: A. seguridad jurídica expresada como la existencia de un marco legal para el desarrollo del sector minero y B. El establecimiento de condiciones competitivas reales en la extracción de minerales para obtener Impuestos, regalías y dar empleo para hacer un adecuado uso del sector minero del país.

- En la confianza legítima: Desarrollar unas condiciones de confianza entre el sector productivo y las comunidades afro-indígenas en sus asentamientos rurales y el gobierno actuando como regulador de la actividad minera.

- En la infraestructura: Generar vías de acceso para la movilización del transporte entre los centros de explotación; seguridad y salud en el trabajo para prevenir la pérdida de vidas humanas producto del irregular manejo de los elementos con los cuales se ejecuta la actividad minera nacional.

- En la información: Divulgar los conocimientos en términos geológicos, mineros, su administración cuidado y manejo de los recursos como soporte técnico. 
- En la institucionalidad minera eficiente: atender las disposiciones establecidas por el Ministerio de Minas y Energía para la autorización y realización de trámites para ejercer la actividad relacionada con la extracción de minerales metalíferos-no metálicos-carbón mineral-extracción de petróleo crudo y gas natural. "En los años noventa, se introdujeron grades reformas en el estado en materia minera para permitir la llegada de mayor inversión extranjera, con el fin de impulsar el desarrollo económico del país a través de una mayor obtención de materias primas, lo que ocasionó un crecimiento importante en los precios de los metales como el cobre y el oro" (Sánchez \& Lardé, 2006, pág. 9). De otro lado, hubo requerimientos por parte del Banco Mundial y el Fondo Monetario Internacional, sumado al crecimiento expansionista de Estados Unidos, China, Japón en la demanda de materias primas en hidrocarburos.

El Estado nunca ha estado preparado para realizar una planificación estratégica adecuada para lograr beneficios, fruto de la actividad minera y como las comunidades afro-campesinas se han visto afectadas con la explotación desordenada. En el departamento del Cauca ya han pasado innumerables episodios de abusos en épocas pasadas sin una adecuada atención del gobierno central: "La minería ilegal es aquella declarada por los gobiernos de turno como toda práctica realizada por fuera de las normas constitucionales y legales dentro del marco legislativo de autoridad competente y la que mayores estragos ha causado en la vida de las personas, especialmente en poblaciones étnicas del pacífico" (Banguera, 2017, página 51).

En el 2005 llega a Timbiquí los cultivos de la hoja de coca y la invasión de la minería ilegal, un grupo de mujeres conformó una red con el fin de garantizar la alimentación de las familias y defender los derechos de la comunidad. "Realmente son pocos los agricultores dedicados al cultivo de la tierra se ven uno o dos, los demás, están en la minería, viven pendientes de barenquear y lo ancestral ha pasado a otro plano". (Andrés García, s.f.). Para garantizar los ingresos las mujeres siembran plantas medicinales y las venden en el casco urbano de la ciudad conjuntamente con alimentos preparados por ellas y otras mujeres se han dedicado a la elaboración de artesanías para mejorar sus ingresos a través de los viajeros en tránsito por estas poblaciones olvidadas. (Juan Diego Restrepo E., 2016)

En Colombia gran parte de la actividad minera tiene lugar en zonas de frontera. Esto significa que las cuencas compartidas se convierten en las principales vías de contaminación por mercurio. La zona más afectada se encuentra a lo largo del rio Caquetá. En el año 2000 el precio del oro era de US \$279.1 el gramo. Doce años después alcanzó un máximo histórico de US \$1.699.0 (Infoamazonía, 2018).

En otros lugares de la geografía colombiana, como Boyacá la guaquería se desarrolla en el occidente de la región en pueblos como: Muzo, Maripí, Pauna, Santa Bárbara, Otanche y San Pablo de Babur las mujeres con sus hijos son las llamadas a asistir esta actividad llamada minería de la cual se alimentan muchas personas en la ilegalidad. Con el hecho de buscar esmeraldas, por un kilate se explotan 10 toneladas de tierra estéril. (Navarrete, 2016).

Las matamberas son mujeres que pertenecen a la red de las 22 organizaciones femeninas comunitarias que trabajan conjuntamente en la región, muchas de ellas, hacen parte de la explotación minera con ingresos mínimos que les dan los interesados en el barenqueo como ayuda para hacerle frente a las adversidades del Cauca (Juan Diego Restrepo E, 2016, pp. 22-24).

En Segovia al nordeste de Antioquia y Remedios se produce el $11 \%$ del oro que se extrae de Colombia cifras de la Agencia Nacional de Minería trabajo realizado por mujeres cabeza de familia desplazadas por la violencia, se dedican a "Chatarrear" (Las Chatarreras como se les llama son caras femeninas de la minería ilegal, pág 38 Ricardo Cruz). El trabajo depende de la voluntad del dueño de la mina y de la multinacional que opera en la zona, con las amenazas de los grupos irregulares que operan en la región. Se trabaja a través de contratos de asociación (tercerización de la explotación minera). Una de ellas comenta: "Se explota el socavón se lleva la mina en bruto a la planta de beneficio de la multinacional y le dicen..., tan solo se produjo 10 gramos de oro; para comprobar la veracidad de lo hablado se lleva el mismo material a un laboratorio para que lo analicen y el 
resultado es de 35 gramos de oro por kilo" (página 45 Ricardo Cruz, las chatarreras, cara femenina de la minería de Segovia).

La minería ilegal, en su mayoría afecta las comunidades indígenas y se ha referido como minería artesanal, a pesar de que, ante los ojos de los gobernantes, es una práctica ilegal: "En Brasil, Colombia, Ecuador, Guyana y Perú los recursos minerales son propiedad del estado, en consecuencia, es el gobierno quien tiene la potestad para decidir sobre sus operaciones y su explotación". (Patricia Quijano, 2020)

A continuación, se presenta la información del caso en estudio se trabaja en la matriz de Vester para identificar las causas que determinan el principal problema de la minería ilegal en Colombia (ver tabla 7).

\begin{tabular}{|c|c|c|c|c|c|c|c|c|c|c|c|c|c|}
\hline & $\mathrm{P}-1$ & $\mathbf{P}-2$ & $\mathrm{P}-3$ & $\mathrm{P}-4$ & $P-5$ & P-6 & P-7 & P-8 & P-9 & P-10 & $\mathrm{P}-11$ & P-12 & INC. \\
\hline \multicolumn{14}{|l|}{ PROBLEMA 1} \\
\hline \multicolumn{14}{|l|}{ La minería ilegal genera una explotación } \\
\hline \multicolumn{14}{|l|}{ inadecuada de los recursos propios de la } \\
\hline Región. & & 2 & 3 & 3 & 2 & 3 & 2 & 3 & 2 & 0 & 0 & 2 & 22 \\
\hline \multicolumn{14}{|l|}{ PROBLEMA 2} \\
\hline \multicolumn{14}{|l|}{ Falta presencia del estado en las } \\
\hline poblaciones donde se ejerce la actividad & 3 & & 3 & 0 & 3 & 1 & 0 & 0 & 0 & 0 & 0 & 2 & 12 \\
\hline \multicolumn{14}{|l|}{ PROBLEMA 3} \\
\hline \multicolumn{14}{|l|}{ Presencia de grupos al margen de la ley } \\
\hline \multicolumn{14}{|l|}{ financian la explotación con dadivas a la } \\
\hline Población. & 3 & 3 & & 0 & 3 & 0 & 0 & 0 & 3 & 0 & 0 & 0 & 12 \\
\hline \multicolumn{14}{|l|}{ PROBLEMA 4} \\
\hline \multicolumn{14}{|l|}{ Desastre social-ambiental afecta la bio- } \\
\hline Diversidad se destruye la capa vegetal. & 3 & 0 & 0 & & 0 & 3 & 0 & 0 & 2 & 0 & 0 & 2 & 10 \\
\hline \multicolumn{14}{|l|}{ PROBLEMA 5} \\
\hline Graves problemas sociales e inseguridad. & 3 & 2 & 3 & 1 & & 1 & 1 & 1 & 3 & 1 & 1 & 1 & 18 \\
\hline \multicolumn{14}{|l|}{ PROBLEMA 6} \\
\hline \multicolumn{14}{|l|}{ Contaminación de fuentes hídricas, } \\
\hline Producen enfermedades en la población. & 3 & 2 & 1 & 3 & 2 & & 2 & 0 & 1 & 3 & 0 & 3 & 20 \\
\hline \multicolumn{14}{|l|}{ PROBLEMA 7} \\
\hline \multicolumn{14}{|l|}{ No se cuenta con infraestructura vial ni } \\
\hline Servicios públicos. & 0 & 3 & 2 & 2 & 1 & 0 & & 0 & 0 & 2 & 0 & 3 & 13 \\
\hline \multicolumn{14}{|l|}{ PROBLEMA 8} \\
\hline \multicolumn{14}{|l|}{ Intervención de compañías extranjeras } \\
\hline \multicolumn{14}{|l|}{ no le dejan mayores beneficios al país y } \\
\hline A la región. & 3 & 2 & 3 & 2 & 3 & 3 & 2 & & 0 & 0 & 0 & 1 & 19 \\
\hline \multicolumn{14}{|l|}{ PROBLEMA 9} \\
\hline \multicolumn{14}{|l|}{ La falta de educación en la población hace } \\
\hline que sean presa fácil. & 3 & 3 & 3 & 1 & 3 & 1 & 0 & 1 & & 0 & 0 & 1 & 16 \\
\hline PROBLEMA 10 & & & & & & & & & & & & & \\
\hline No existe infraestructura médico hospi- & & & & & & & & & & & & & \\
\hline
\end{tabular}




\begin{tabular}{|c|c|c|c|c|c|c|c|c|c|c|c|c|c|}
\hline talaria para la atención de su población. & 0 & 2 & 1 & 0 & 0 & 3 & 3 & 2 & 0 & & 0 & 0 & 11 \\
\hline \multicolumn{14}{|l|}{ PROBLEMA 11} \\
\hline \multicolumn{14}{|l|}{ La falta de tecnología a nivel académico } \\
\hline en los colegios. & 0 & 0 & 0 & 0 & 0 & 1 & 1 & 2 & 3 & 0 & & 1 & 8 \\
\hline \multicolumn{14}{|l|}{ PROBLEMA 12} \\
\hline \multicolumn{14}{|l|}{ No existe un plan de explotación agrícola } \\
\hline \multicolumn{14}{|l|}{ por parte del Estado para incentivar la NO } \\
\hline \multirow[t]{2}{*}{ Dependencia del cultivo de coca. } & 0 & 3 & 3 & 3 & 1 & 0 & 1 & 1 & 1 & 0 & 0 & & 13 \\
\hline & & & & & & & & & & & & & 174 \\
\hline DEPENDENCIA & 21 & 22 & 22 & 15 & 18 & 16 & 12 & 10 & 15 & 6 & 1 & 16 & 174 \\
\hline
\end{tabular}

Tabla 7. Matriz de Vester caso: explotación minera en Timbiquí (cauca). Fuente: Elaboración propia.

Como se aprecia en los resultados de la matriz de Vester el nivel de incidencia entendida como: como lo que sobreviene en la explotación inadecuada de los recursos naturales propios de la región del Cauca, particularmente en la minería ilegal, se obtuvo un puntaje de 22 puntos. Como nivel de dependencia de este problema se atribuyen dos factores o situaciones a saber: La falta de presencia del Estado en las poblaciones donde se ejerce esta actividad con programas sociales orientados a la población para corresponder a las condiciones humildes de la comunidad compuesta por población afro-indígena-campesina y los precarios servicios de salud y educación e infraestructura física de sostenimiento de sus habitantes. En cambio, hay presencia de grupos al margen de la ley que se aprovechan de la idiosincrasia y la carencia de medios para otorgar dádivas a la gente para que se dediquen a explotar los recursos de una región rica en naturaleza, pero en total abandono por parte del Estado.

\section{Problemática social}

A La minería ilegal genera una explotación de recursos y personas

B Falta presencia del estado

C Presencia grupos al margen de la ley

D Desastre Social-Ambiental en la biodiversidad

E Problemas sociales e Inseguridad

F Contaminación fuentes hídricas

G No cuentan con servicios públicos

H Intervención de Compañías Extranjeras

I Los jóvenes son presa fácil de la explotación minera

J No existe infra estructura Médico Hospitalaria en Timbiquí

K Falta de tecnología a nivel académico en los colegios

L No existe plan de explotación agrícola por parte del estado 


$\begin{array}{rr}\mathbf{X} & \mathrm{Y}=\text { INCIDENCIA } \\ 22 & 2 \\ 12 & 2 \\ 12 & 2 \\ 10 & 15 \\ 18 & 18 \\ 20 & 16 \\ 13 & 1 \\ 19 & 10 \\ 16 & 15 \\ 11 & \\ 8 & 16 \\ 13 & \end{array}$

$$
\mathrm{Y}=\text { incidencia }
$$

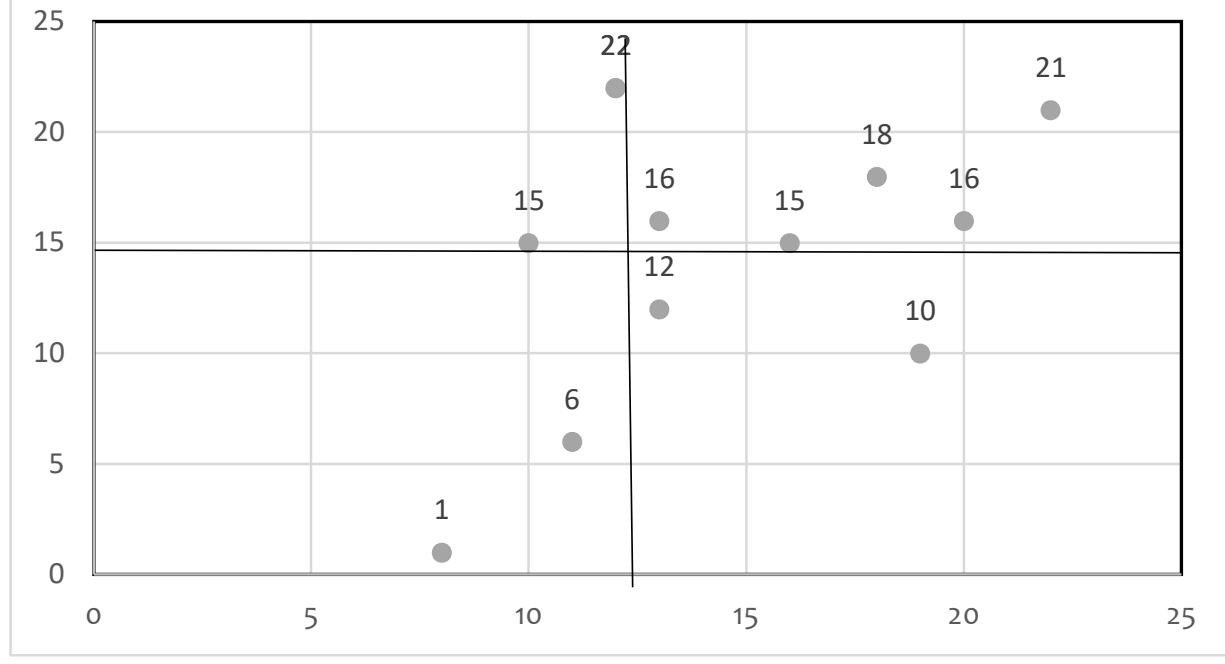

16

Figura 3. Problemática social y análisis de incidencia. Fuente: Elaboración propia.

La minería en Colombia empezó a ser una actividad productiva del sector primario de gran importancia para la nación, con la llegada de los Españoles al territorio nacional y su posterior conquista con las técnicas empleadas en esa época eran más eficientes, por lo cual se permitió explorar más recursos minerales en un menor tiempo, esta actividad se encuentra actualmente en pleno auge trayendo consigo muchas consecuencias de índole político, económico, social y ambiental.

En el siglo XIX la explotación ilícita de oro se estableció como una actividad económica de grandes proporciones en Colombia. Desde esta época nuestro país, ha sido sometido a la explotación de sus minerales y la aprobación de leyes que favorecen la actividad humana. Esta tendencia al aumento de la explotación legal ha evolucionado en paralelo con la explotación ilegal (ver figura 4).

En cuanto al aspecto político en el sector de la minería Colombia reglamento y dio a conocer su nueva política de minería en el año 2016 mediante la resolución 40391, A través de esta resolución, el Ministerio de Minas y Energía, adopta y compila las diferentes políticas y lineamientos de política existentes en el país. Se da la creación de un documento llamado "La Política Minera Nacional” de la República de Colombia.

Colombia es un país que ha desempeñado labores de minería desde hace muchos años, pero debido al cambio que ha tenido el sector minero con respecto al tema ambiental, a la explotación ilegal de recursos minerales y a diversos factores se ve en la necesidad de crear y definir una serie de estrategias orientadas a hacer más competitiva y productiva esta industria.

En el aspecto económico el negocio del oro en el 2017 presentó un comportamiento muy particular, tanto en producción como en exportación, que llamó la atención de los expertos en la operación aurífera del país. Mientras la producción del metal precioso decreció con respecto al volumen registrado en el 2016, paradójicamente sus exportaciones crecieron entre un año y otro. Datos de la Agencia Nacional de Minería (ANM), indican que en el 2017 la producción de oro reportada por las mineras fue de 41 toneladas, es decir 20 menos a la extracción del 2016 que fue de 61 toneladas. Estas cifras contrastan con los reportes del Departamento Administrativo Nacional de Estadística (DANE) en cuanto a exportaciones, ya que para el 2017 estas fueron de 58 toneladas, 10 más que en el 2016, cuyo volumen reportado fue de 48 toneladas, es decir, que ese $20,2 \%$ de crecimiento en los mercados internacionales le representó al país un aumento en ventas por US\$245 
millones. Según el DANE, en el 2017, el país percibió por la comercialización del metal precioso US\$1.806 millones ( $\$ 5,1$ billones), frente al 2016 que fue de US\$1.561 millones ( $\$ 4,45$ billones).



Figura 4. Mapa de la minería ilegal en Colombia. Fuente: Recuperado de: https://www.elmundo.com/noticia/Extraccion-ilegal-o-el-dinero-porsobre-todas-las-formas-de-vida/376594https://www.elmundo.com/noticia/Extraccion-ilegal-o-el-dinero-por-sobre-todas-las-formas-devida/376594

En el aspecto social las poblaciones vulnerables se ven afectadas negativamente, específicamente cuando se realizan actividades mineras en territorios designados para ellas y en Colombia a pesar de ser minorías existen en un número significativo. Según datos del censo del año 2005 (DANE, 2005), en Colombia habitan 84 pueblos indígenas, distribuidos en 704 territorios colectivos conocidos como resguardos, en tanto que la población afrodescendiente supera el 10\% de la población nacional, con más de 150 territorios colectivos de comunidades negras tradicionales en la región del Pacífico. Además, existen comunidades ROM o gitanas, hay aproximadamente 60 lenguas nativas y las comunidades campesinas siguen estando presentes en todo el territorio nacional (Ministerio de Cultura, 2013). Un corolario más: en Colombia hay 35 pueblos indígenas en peligro de extinción física y cultural ante los cuales la Corte Constitucional le ordenó al Estado tomar medidas urgentes a su favor, como el caso de las etnias Jiw y Nükak, asentados en los departamentos de Meta y Guaviare. Entre los factores de riesgo reconocidos por la ONIC está el desarrollo de las industrias extractivas en el territorio de estas comunidades, que por su alta vulnerabilidad socioeconómica son fácilmente cooptadas por empresas para contar con su aceptación a la entrada de megaproyectos.

En el aspecto ambiental la actividad minera produce prosperidad en las zonas donde se lleva a cabo, pero indudablemente tiene un impacto nefasto en el tema ambiental a saber:

1. Daño a la tierra.

2. Liberación de sustancias tóxicas. 
3. Drenaje acido de minas.

4. Salud y seguridad en el trabajo.

A continuación, se presenta la matriz de los interesados para el caso de explotación de oro en la población de Timbiquí del Departamento del Cauca:



Tabla 8. Matriz de los interesados caso Timbiquí, Cauca. Fuente: Elaboración propia.

A este análisis se suma el que se plantea en el árbol de problema que se presenta en la figura 5: 


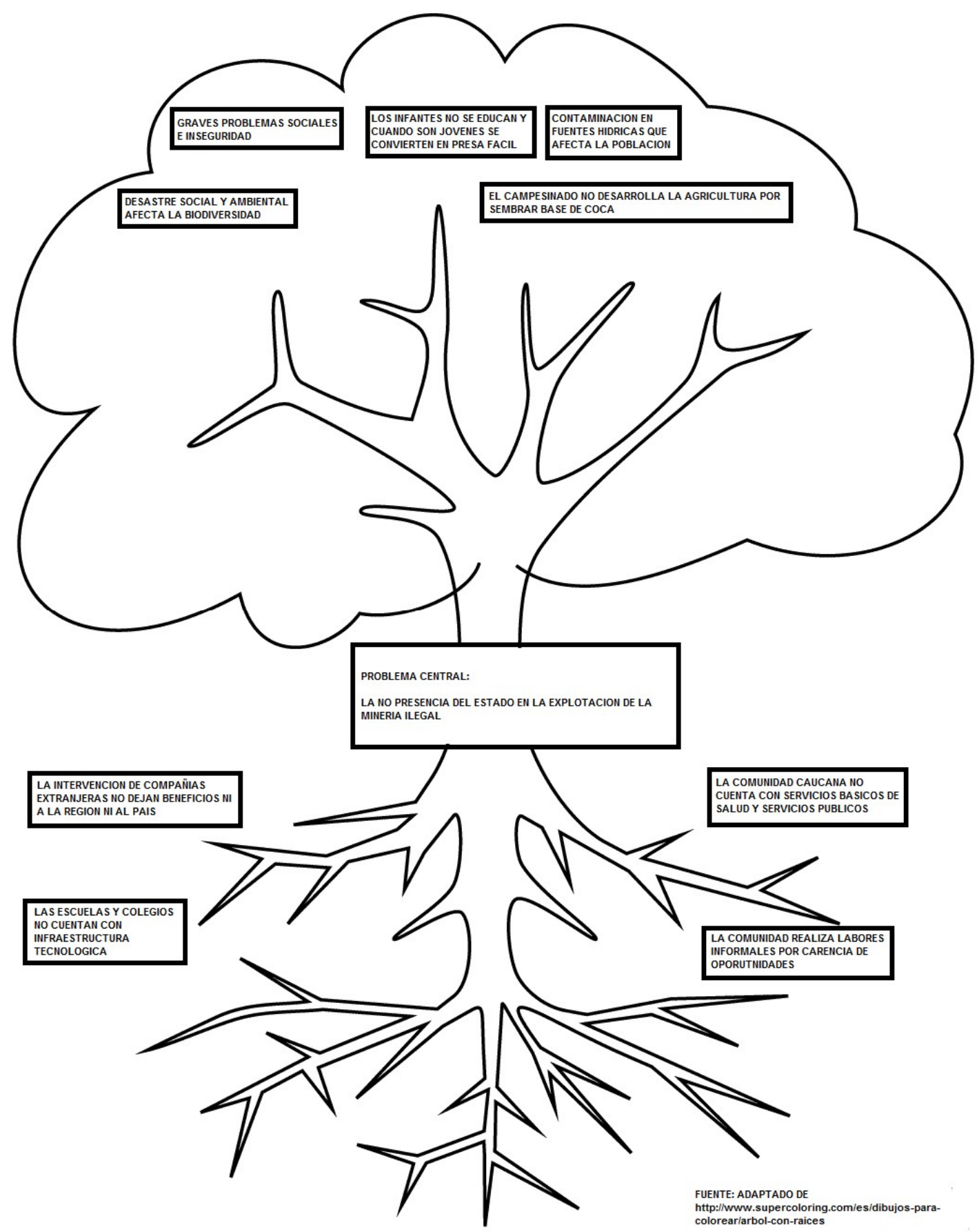

Figura 5. Árbol de problema Caso Tmbiquí, Cauca. Fuente: Elaboración propia. 


\section{FORMULACIÓN DE UN PLAN DE ACCIÓN}

¿Qué se va a hacer? Concertar con los ministerios del Medio Ambiente, Hacienda, Justicia, Educación, Trabajo, Agricultura, Salud y Minas - Energía y con la ayuda del Presidente de la República y la Ministra del Interior la elaboración de un programa para la gobernación del Cauca en el cual se establezca el direccionamiento estratégico del plan ambiental para la región (sostenibilidad -protección de los recursos naturales); se calcule los recursos económicos para dicho programa y de donde se pueden obtener para ponerlo en marcha; Se apliquen las leyes que salvaguarden la población y se judicialice los grupos que invaden la región y generan desorden, corrupción y mal aprovechamiento de la minería ilegal; se establezca un programa educativo con la juventud en apoyo tecnológico para lograr emprendimientos económicos y economía naranja gratuito, para las poblaciones afectadas. Se formalice el trabajo informal para que las mujeres logren el sustento para sus hijos; se diseñe un programa de cultivos propios de la región para el campesinado y la población indígena de la región con apoyo del sector empresarial. Se organice el sistema de salud con asocio de la organización Panamericana de la Salud para lograr mejor infraestructura hospitalaria y se le ponga fin a la minería llegal en el Departamento.

¿Cómo se va a hacer? A través de mesas de trabajo por programa en donde participe la comunidad y los dirigentes de la región. Presentación de talleres donde se imparta ética social y competencias ciudadanas. Mostrar al país con una campaña difusora que en Colombia si hay presencia del Estado para cumplir su función de regulador y protector del territorio nacional.

¿Cuándo se va a hacer? Se deberá elaborar un Diagrama de Gantt de acuerdo al resultado de las mesas de concertación ministerial se determinen objetivos; actividades; tiempos y recursos.

¿Dónde se va a hacer? A nivel macro en el departamento del Cauca, a nivel micro en las zonas afectadas por la minería ilegal.

¿Quiénes participarán? Líderes comunitarios y gerentes sociales de proyectos quiénes tendrán la responsabilidad de dar cumplimiento a las premisas formuladas y diseñadas para su ejecución.

¿Cuánto vale lo que se va a hacer? Programa de inversiones + Financiamiento y presupuestos de operación para todo el departamento.

\section{CONCLUSIONES}

La minería ilegal viene en crecimiento año tras año en Colombia, el incremento para el 2019 es de 340 municipios afectados en la explotación aurífera, producto del descuido y desatención por parte de los organismos del estado ya que no desarrollan la función de regulación y protección de los recursos naturales. El año inmediatamente anterior los municipios con minería ilegal eran 317.

Para la Gestión Gerencial existen 14 competencias directivas que requieren de ser practicadas por los hábiles gerentes para obtener los resultados esperados en una Gestión de Proyectos Sociales, en donde se involucran los recursos naturales y los demás capitales implicados, las personas de una región, los gobernantes y políticos en el juego de voluntades e intereses, unas a favor y otras en contra en las acciones de un proyecto comunitario.

La utilización del árbol problema con el apoyo de los resultados de la matriz de Vester, permitió aproximarse al problema central del caso en estudio, las causas y consecuencias derivadas de las acciones incorrectas y oportunismos de quienes se burlan de la ley con el único objetivo de buscar beneficios particulares y posiciones egoístas sin importar las personas residentes quienes sufren la afectación permanente. 
El diseño de un plan de acción estratégico por parte de los interesados en buscar que el trabajo y los beneficios sean de pensar en ganar/ganar; (Covey S, los 7 hábitos de la gente altamente efectiva), permite que el proceso funcione, los acuerdos surgen a partir de las relaciones. Los resultados deben ser productos deseados con políticas y medidas evaluadas para alcanzar logros donde toda la comunidad, las empresas y el estado salgan favorecidos de las decisiones por la acción conjunta.

La matriz de los interesados muestra los actores que intervienen en la situación y clarifica las posiciones antagónicas a trasvés de sus intereses y actitudes, permite reconocer quiénes participan y hasta donde el poder puede ejercer influencia en las personas y conocer de qué tipo, dependiendo de sus alcances.

Un proyecto de Gerencia Social implica de la adopción de posturas éticas por parte del estado, las compañías extranjeras y la comunidad donde los impactos generen desarrollo Económico, Social, Humano y Ambiental (sostenibilidad de los recursos). En este sentido "La gerencia Social es un conjunto de elementos conceptuales, metodológicos e instrumentales destinados a lograr una eficaz y eficiente combinación de recursos humanos, físicos, tecnológicos y financieros que garanticen los planes, programas y proyectos en beneficio de las personas, familias, grupos, organizaciones y el Estado. (Quintero, 2010).

\section{REFERENCIAS BIBLIOGRÁFICAS}

Ajenjo Domingo A. (2000) Dirección y Gestión de Proyectos (un enfoque práctico). Rama Editorial, Madrid (España). Pág.: $2-26$

Ansoff H. Igor \& Mc Donell Edward (1997) la Dirección Estratégica en la Práctica Empresarial $2^{\mathrm{a}}$ Ed. Addison Wesley Iberoamericana U.S.A.

Banguera B. Aldo B (2017) Prácticas Mineras y Cambio Socio-Cultural en el corregimiento de San José (Municipio de Timbiquí) Universidad Del Pacífico. Facultad de Humanidades y Bellas Artes, programa de Sociología. Páginas 143.

Chung, P (2009) Prospectiva estratégica más allá del plan estratégico. Revista de la Facultad de Ingeniería Industrial, 12 (2), 27 - 31

Constitución Política de Colombia. Año 2003. Momo Ediciones

Cruz Ricardo (2016) Las chatarreras cara femenina de la minería en Segovia (Antioquia). Recuperado de: https://verdadabierta.com/especiales-v/2016/mujeres-guerra/segovia-mujeres-minasoro.html\#: :text=En\%20Segovia\%20y\%20Remedios\%2C\%20municipios,que\%2olos\%2omineros\%20han\%20 desechado

De Ansorena Cao, Álvaro (1996) 15 pasos para la selección de personal con éxito (método e Instrumentos) $1^{\text {a }}$ Ed. Paidos. Sant Andria de Basos Barcelona (España).

Edvinsson L. \& Malone M (1999) El capital intelectual, Editorial gestión 2000. Barcelona (España).

García Andrés (2016) Matamberas la red que sostiene la identidad afro de Timbiquí (Cauca)

González L (2013) Impacto de la minería de hecho en Colombia. Estudio de caso: Quibdó, Istmina; Timbiquí; López de Mi cay; Guapi; El Charco; Santa Bárbara. Instituto de estudios para el desarrollo y la paz INDEPA. Pág.4-5.

Ilpes (Instituto Latinoamericano De Planificación Económica y Social) 15 Ed. Siglo veintiuno Editores.

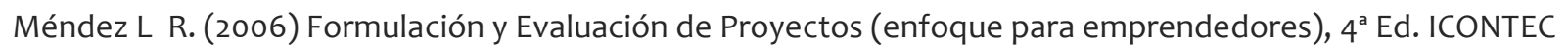


Motta Paulo Roberto (1993) La Ciencia y el Arte de ser Dirigente $1^{\mathrm{a}}$ Ed. Tercer Mundo Editores; Ediciones Uniandes. Bogotá D.C., Colombia.

Navarrete Tatiana (2016) Mujeres víctimas silenciosas de la esmeralda en Boyacá.

Quinteros Camacho J \& Hamann Pastorino A (2017) Planeamiento estratégico Prospectivo (métodos Mactor y Smic. $1^{a}$ Ed. Fondo Editorial ECOE Ediciones. Pág. 16 - 56.

Quintero Víctor M. (2010) Impacto Social. (Evaluación de Proyectos de Desarrollo), $1^{\text {a }}$ Ed. Impresora Feriva S.A., Cali (Colombia).

Restrepo E Juan D. (2016) Verdad Abierta.com Mujeres tras el telón de la guerra. Recuperado de: https://verdadabierta.com/especiales-v/2016/mujeres-guerra/index.html

Steiner A. George (1997) Planeación Estratégica (Lo que todo director debe saber): 22 Ed. Compañía Editorial Continental S.A. de C.V. México. 